University of Nebraska - Lincoln

DigitalCommons@University of Nebraska - Lincoln

Papers in the Earth and Atmospheric Sciences Earth and Atmospheric Sciences, Department

\title{
$9-2019$
}

\section{Integrated Imaging: A Powerful but Undervalued Tool}

Irina Filina

Ed K. Biegert

Luise Sander

Victoria Tschirhart

Neda Bundalo

See next page for additional authors

Follow this and additional works at: https://digitalcommons.unl.edu/geosciencefacpub

Part of the Earth Sciences Commons

This Article is brought to you for free and open access by the Earth and Atmospheric Sciences, Department of at DigitalCommons@University of Nebraska - Lincoln. It has been accepted for inclusion in Papers in the Earth and Atmospheric Sciences by an authorized administrator of DigitalCommons@University of Nebraska - Lincoln. 
Authors

Irina Filina, Ed K. Biegert, Luise Sander, Victoria Tschirhart, Neda Bundalo, and Cara Schiek-Stewart 
Published in The Leading Edge 38:9 (September 2019), pp. 720-724; doi: 10.1190/tle38090720.1

Copyright (C) 2019 Society of Exploration Geophysicists. Used by permission.

Published online September 4, 2019.

\title{
Integrated Imaging: A Powerful but Undervalued Tool
}

\author{
Irina Filina, ${ }^{1}$ Ed K. Biegert, ${ }^{2}$ Luise Sander, ${ }^{3}$ Victoria Tschirhart, ${ }^{4}$ \\ Neda Bundalo, ${ }^{5}$ and Cara Schiek-Stewart ${ }^{6}$
}

1. University of Nebraska-Lincoln, Lincoln, Nebraska, USA

2. Houston, Texas, USA

3. Sander Geophysics, Ottawa, Ontario, Canada

4. Geological Survey of Canada, Ottawa, Ontario, Canada

5. Anadarko Petroleum Corporation, The Woodlands, Texas, USA

6. Shell Exploration and Production Company, Houston, Texas, USA

Corresponding author - Irina Filina, email ifilina2@unl.edu

\begin{abstract}
Following the 2018 SEG Annual Meeting, the Gravity and Magnetics Committee held a postconvention workshop titled "Integrated Imaging." The half-day workshop attracted nearly 50 participants from various backgrounds. Three primary objectives of the workshop were to explore the nonseismic toolbox, highlight real examples of integrated projects that benefited (or did not benefit) from nonseismic data, and provide geoscientists from all backgrounds a learning opportunity to see how they might optimize the value of their imaging projects via integration with relatively low-cost nonseismic methods. The workshop had a highly interactive format that differed from traditional presentation-based settings. After eight brief case studies were presented, three concurrent guided discussions ensued. Participants were divided into three groups, and each group focused on one discussion topic at a time. The groups rotated, allowing everyone to discuss all three topics. The first discussion was centered on two general questions: what is integrated imaging and what tools are available for it? The second discussion provided an opportunity to examine the relationships between different physical properties that must be managed during integrated multiphysics analysis. The third discussion focused on the costs and benefits of a multiparameter data acquisition. According to feedback from participants, these discussions were the most valuable part of the workshop. The participants agreed that an integrated approach in geophysical data analysis is a powerful but
\end{abstract}


currently undervalued tool. Also noted were the value of integration with nonseismic methods illustrated in the case studies and the need for the integrated approach in data analysis to be taught in schools in addition to the classic overview of individual geophysical methods.

\section{Introduction}

Cost-effective imaging of the subsurface requires rapid integration of different geophysical measurements with geologic control and insights to generate actionable information. Multimodal, multiscale data sets constrained by geology can speed up the cycle time, reduce cost, and improve confidence in our images and interpretation of the subsurface. The combined analysis of multiple geophysical data sets was a theme of the postconvention workshop titled "Integrated Imaging," organized by the Gravity and Magnetics Committee for the 2018 SEG Annual Meeting in Anaheim, California. The key questions posed in the workshop were:

- Are we using the right tools and data sets to solve problems?

- Do we understand why and how to use those tools?

- Can we improve images, optimize our workflows, and provide results efficiently at lower cost?

Nearly 50 participants from industry, academia, and the mining community participated in this workshop, the leaders of which utilized a rather nontraditional format. Instead of a series of presentations like one might see in a typical conference session, most of the workshop was dedicated to interactive discussions. To set up those discussions, the workshop opened with a series of eight brief talks (limited to five minutes each) illustrating how the integration of several geophysical methods influenced the resultant geologic models. The case studies focused on integrated imaging in various geologic settings for a wide range of problems, such as improving the salt model via integration of seismic with gravity and magnetics, determining maturity of unconventional plays, or delineating mineral deposits with joint analysis of multiple geophysical data sets. Several talks were devoted to new advances in methodologies, such as joint inversions, Kirchhoff migrations of timedomain electromagnetic (TEM) data, and machine learning algorithms that can be used for integrated analysis.

Figure 1 shows a collage from the notes taken during guided discussions. Workshop participants quickly agreed that, when interpreted individually, each and every type of geophysical data allows multiple interpretations and that a key driver for the inclusion of more than one type of geophysical data in the analysis is to reduce that interpretation ambiguity. Gravity and magnetic fields integrated with other geophysical data can be used throughout the exploration life cycle to address issues of source rock deposition, source maturation, hydrocarbon migration, reservoir prediction, trap, vertical seal, and timing (see Gibson and Millegan, 1998; Johnson, 1998). Case studies in the published literature have shown that including gravity and gravity gradient data in a seismic imaging workflow often can improve the seismic image dramatically; that gravity, magnetic, and reflection seismic data together can be used to reduce ambiguity about the lithology (rock properties) 
of imaged structures; that magnetic, magnetotelluric, and seismic refraction data can improve images of deep structures; that electromagnetic, reflection seismic, gravity data, and magnetotelluric data together can be used to improve the image of reservoirs, reduce structural uncertainty cum ambiguity, and infer fluid type; and that adding heat flow data to ambiguous joint interpretation of seismic, gravity, and magnetic data can eliminate certain structural interpretations.

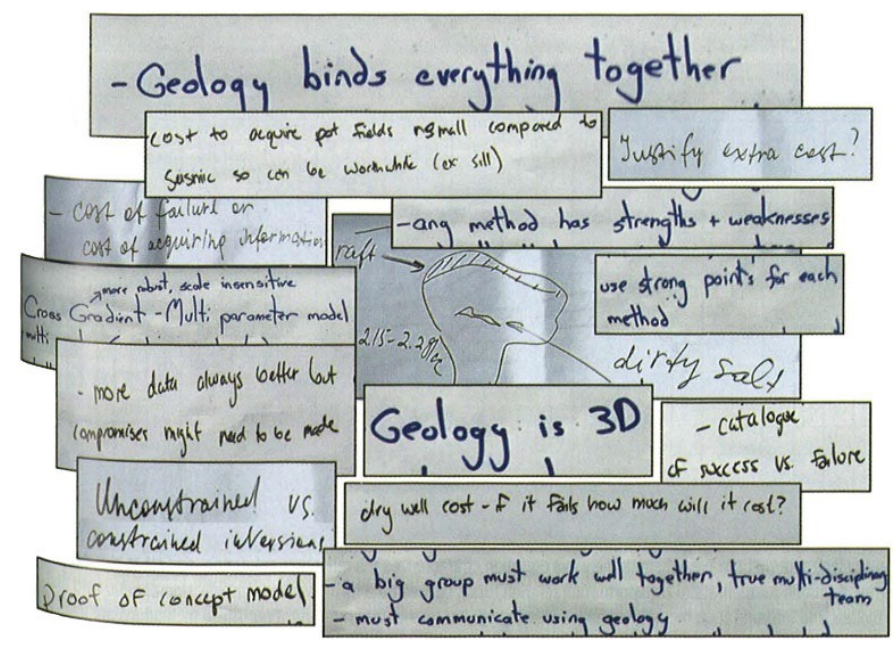

Figure 1. A collage of notes taken during guided discussions.

The inherent ambiguity of any single geophysical measurement usually can be mitigated by including one or more different data modalities that either measure complementary properties of the earth relevant to the problem being investigated or that employ data acquisition techniques that illuminate the target differently. The ability of integrated imaging, joint integration, and multimodal inversion to reduce interpretational ambiguitythat is, to make better-informed decisions and to add value-was the primary focus of the discussions that were scheduled after the case studies. Workshop participants were split into three groups to rotate through three guided discussions. The topic of each discussion was predefined, and the facilitator was guiding the discussion as well as taking notes. The key points out of these three discussions are summarized in the following.

\section{Integrated imaging}

The first discussion was focused on defining integrated imaging and identifying the tools we have to do it. Extending Sheriff's (2002) definition of imaging to all types of geophysical data, we understand imaging to be the inversion of data to an estimate of subsurface distribution of a physical property; a nonunique process. Integrated imaging encompasses the inversion of multiple geophysical data sets of different modalities or of different physical properties to produce a harmonious description of the subsurface properties. 
The overall objective of the integrated analysis as formulated by the participants was to derive the best geologically sound subsurface model that fits/honors/agrees with a multitude of complementary geophysical data sets and assumptions about the earth. The point was argued that no amount of data (without taking a physical sample) can ever prove a hypothesis or interpretation correct; rather, data can only disprove a hypothesis (Popper, 2003). Thus, the addition of complementary data can greatly reduce interpretational ambiguity, even if a single model cannot be unambiguously determined.

The wide spectrum of inversion methodologies currently used for integrated analysis was discussed during the workshop. These include basic unconstrained inversions known to be highly dependent on the starting model and which generally should be followed with a constrained analysis. The integration assumes the use of several geophysical data sets, so either cooperative inversions or more sophisticated joint inversion algorithms generally are used. The latter may involve various mathematical stabilizers enforcing various assumptions of the interpreter, such as correlation between different physical properties, or the sharpness of edges in the model, etc. These additional assumptions represent a priori information that reduces the ambiguity of the interpretation or, equivalently, reduces the null space of the inversion.

However, the participants agreed that there are no truly unconstrained inversions, as some common-sense constraints always should be enforced, such as the upper/lower boundaries on physical properties of the modeled rocks or some limitations on the depth of the layers in the model, such as bathymetry, etc. Those "unconstrained" inversions are dependent on the starting model and do not resolve the model unambiguously. Despite that, some participants stated that these "unconstrained" inversions can serve as a quick tool for the initial evaluation of the data as they outline the general scope of variations in the subsurface model that are required to fit the observed geophysical signal.

The constrained individual inversions (that use geologic constraints with one geophysical data set, such as seismic, gravity, magnetics, or TEM) can be used to evaluate the sensitivity to various model parameters. These are useful to test if the model might be resolved to the desired extent with a single given geophysical data set. Cooperative inversions, in which the output of the individual inversion of one method (say, gravity) serves as a starting model for the individual inversion of the other method (say, magnetics), anticipate the agreement of the final result with both geophysical data sets. The most complex case, the joint inversion, in which two or more geophysical data sets are inverted simultaneously, usually utilizes all available geologic constraints. Depending on the assumptions and formulations of the constraints, these joint inversions may depend on some sort of correlation either between physical properties of the rocks in the model, such as densities, magnetic susceptibilities, velocities of seismic waves, resistivities, etc., or between their correlative gradients.

Two exciting recent tools were discussed in the workshop. Gramian constraints and joint focusing stabilizers were used in the joint inversion of gravity, magnetic, electromagnetic, and seismic field data, and the Gramian method was also used for a stable inversion for the 3D magnetization vector in the case of remanent magnetization leading to an impressive imaging result for a geothermal application in Japan (Zhdanov, 2018). A unique mathematical transformation relating the diffusive transient electromagnetic field and a 
virtual wavefield enables the use of the seismic imaging toolbox (see Fig. 2 for an example using Kirchhoff migration) and interpretation techniques for transient electromagnetic data interpretation (Qi et al., 2018). This methodology can be used in electrical resistivity imaging applications, including near-surface seismic statics, karst detection, unexploded ordnance detection, tunneling, shallow gas sands, aquifer detection, ore bodies, hydrocarbon detection, detecting drilling hazards, and due diligence surveys.

a

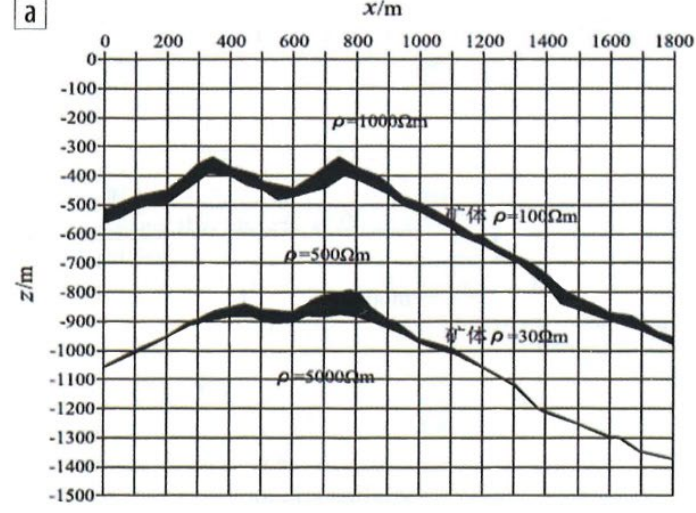

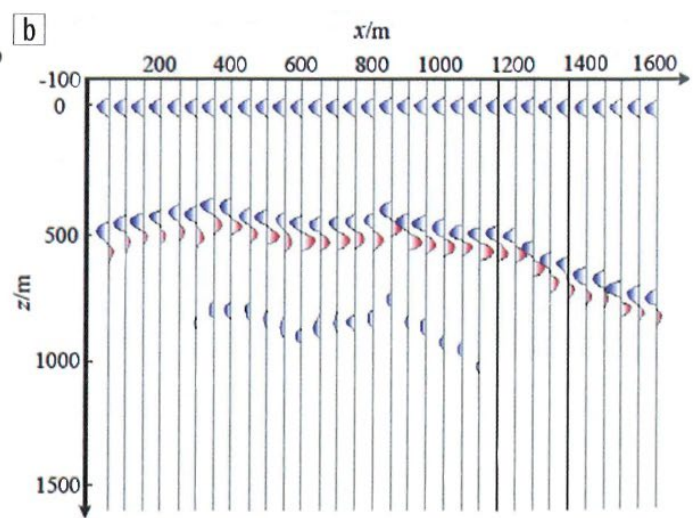

Figure 2. The example of utilizing Kirchhoff migration for TEM data from Qi et al. (2018). (a) Geologic model. (b) TEM virtual wavefield. The diffusion equation in electromagnetics characterizes the induction characteristics of a low-frequency electromagnetic wave, whereas the wave equation characterizes the reflection, refraction, and other propagation features of a wavefield and is the basis for wavefield imaging. A unique mathematical transformation relation exists between the diffusive transient electromagnetic field and a virtual wavefield enabling the use of the seismic imaging toolbox and interpretation techniques for transient electromagnetic data interpretation. These techniques are used in electrical resistivity imaging applications, including near-surface seismic statics, karst detection, unexploded ordnance detection, tunneling, shallow gas sands, aquifer detection, ore bodies, hydrocarbon detection, detecting drilling hazards, and due diligence surveys.

\section{Rock properties}

The subject of the second discussion was the relationships between different physical properties that must be managed during integrated multiphysics analysis. Participants agreed that rock properties are key model parameters for integrated imaging. The best way to constrain them is to use well logs. If no well data are available, the rock properties of the model can be assigned based on physical property measurements on drill core samples or outcrops and, if all else fails, on published compilations. In the case of a joint inversion when two (or more) geophysical data sets, say gravity and magnetic data, are inverted simultaneously, the relationship between the corresponding physical properties (in this case density and magnetic susceptibility, respectively) needs to be understood or derived from the data. The participants agreed that the correlations between various physical properties must agree with the mapped and/or logged geology. Numerous empirical relationships between rock physical properties exist, such as various relationships between density 
and seismic velocity, but they should be validated against local geology in each case. Some practical methods that ensure crosscorrelation between various physical properties or the structure of bodies were also discussed, such as using the cross-gradients stabilizer during multimodality joint inversions. The Bayesian approach can be utilized to establish the relationships between different physical properties from the well logs as well as to evaluate the uncertainty of the resultant model.

\section{Value of integrated imaging}

The third discussion was dedicated to the overall value of the integrative analysis. In terms of the cost of nonseismic geophysical data sets, the participants noted that potential field data are significantly less expensive to acquire, process, and interpret than seismic data. However, potential field data are often perceived to hold less value due to their inexpensive cost contrary to numerous case histories illustrating how they actually reduce exploration cost. Biegert and Millegan (1998) enumerate more than 25 case histories in which potential field data added value to exploration projects, while Blaizot and Montagnier (2013) provides examples of Total's use of nonseismic technologies (gravity, gravity gradiometry, magnetotelluric, etc.) to improve imaging, especially prestack depth migration, in rift systems, subsalt exploration, presalt carbonates, abrupt margins, large deltas, and foothills. Colombo et al. (2010) demonstrate how seismic imaging problems caused by complex near-surface conditions can be significantly resolved with the judicious use of gravity. Gravity gradiometry has added value in a number of 3D subsalt seismic imaging problems (for a few examples, see Dejong et al., 2001; Barraud et al., 2010; Dyer, 2011) as well as marine magnetotellurics (Key et al., 2006). The case study of Filina et al. (2015) shows how integration of seismic and gravity results in more confident salt geometry. The example from the Gulf of Mexico presented at the workshop (Yalamanchili et al., 2018; Fig. 3) illustrates the benefits of gravity in discriminating between two equally viable seismic picks for the base of allochthonous salt. Integrating seismic, magnetic, and gravity data in the offshore Eastern Mediterranean allowed high-resolution imaging of salt domes in this tectonically complex and difficult-to-image region (Abbas et al., 2018). Nonseismic methods also can provide more efficient and cost-effective ways to evaluate and derisk unconventional plays (Edman et al., 2015). As presented in this workshop, high-resolution aeromagnetic data were used to map small intrusive bodies and were integrated with core descriptions, geochemistry, and geologic mapping to delineate areas of increased maturity in the Delaware Basin unconventional play (Rauch-Davies, 2018). 

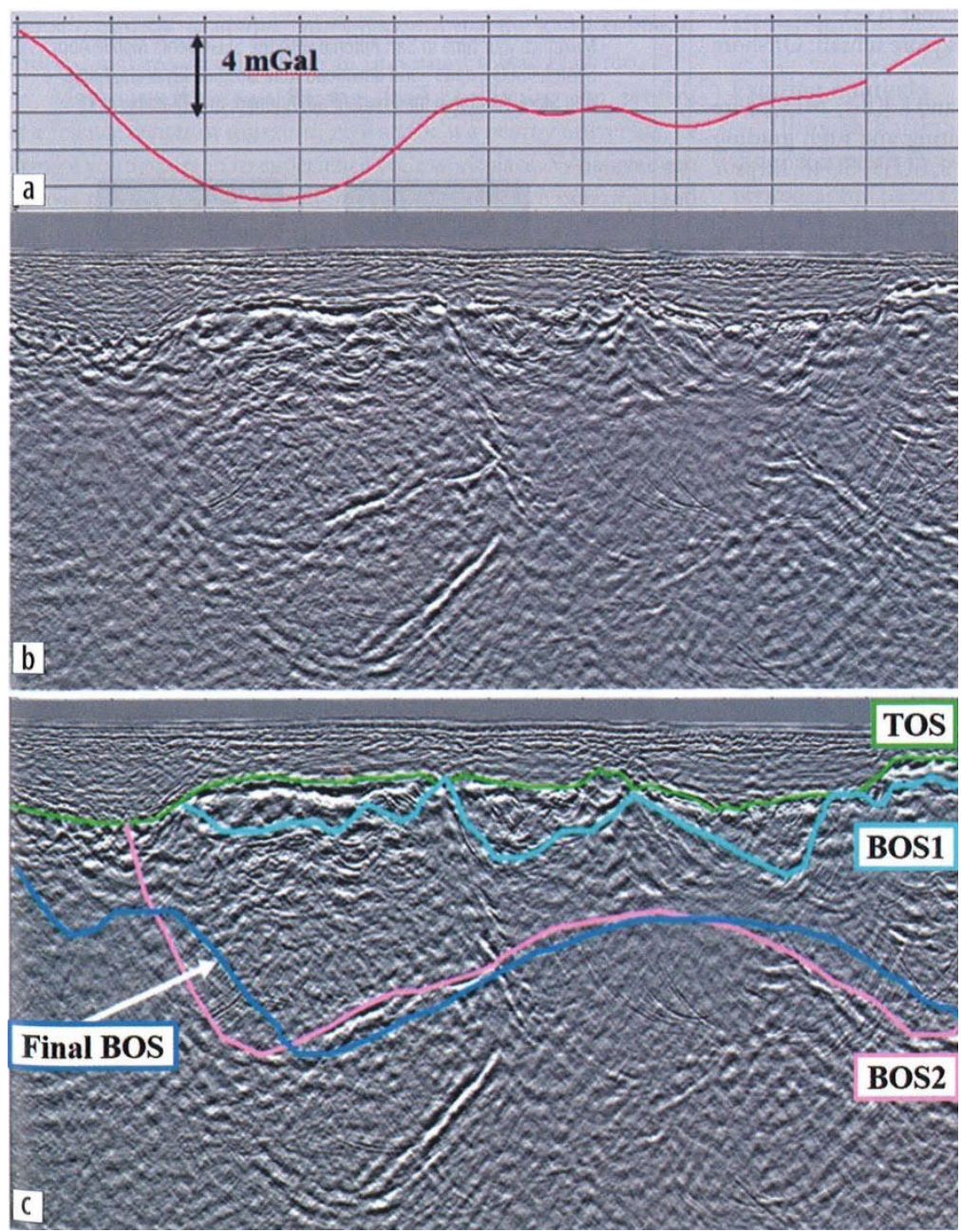

Figure 3. The example of integrated seismic and gravity study in the Gulf of Mexico from Yalamanchili et al. (2018). (a) Gravity anomaly. (b) Uninterpreted seismic section. (c) Seismic interpretations. TOS: top of salt. BOS1 and BOS2: two alternative base-of-salt picks from seismic only. Final BOS shows the base of salt interpreted from integrative analysis of gravity and seismic data.

In each case, the costs of making a wrong decision, reducing uncertainty, and increasing the probability of success need to be assessed as an alternative to the acquisition of an additional geophysical data set. Participants agreed that feasibility studies are the key to evaluate if the model can be resolved with any particular geophysical method and also noted that a downturn in business activity may be an opportune time to showcase nonseismic or integrated results. The participants concluded that a catalog of success and failure cases would be useful to demonstrate the effectiveness of integrated analysis. Moreover, this catalog could serve to overcome the general perception that nonseismic geophysical methods are not worthy investments. The case studies presented at this workshop and the discussions afterward clearly demonstrated the value of integration with nonseismic 
methods. When several different geophysical data sets are jointly analyzed and the proper geologic constraints are enforced, the resultant model usually has a rather narrow range of solutions.

\section{Conclusions}

Integrated imaging implies a combined analysis of multiple geophysical data sets to find the best geologically valid model to fit all available geophysical data sets. Workshop participants concluded that the best integrated imaging results from the effort of a multidisciplinary geoscience team communicating via geology. Since each data set has its own ambiguities, strengths, and weaknesses, the results of each should be evaluated for the given geologic settings, and the strong points from each methodology should be integrated. It is important to have a good understanding of the physical properties when interpreting different data sets. Although there may be areas of no correlation between some different physical properties, separate inversions displayed concurrently can be valuable. It is also important to remember that geology is 3D, and different data may be influenced by geology at different depths and different scales of resolution. The participants agreed that the integrated approach in data analysis should be taught in schools in addition to the classic overview of individual geophysical methods.

The overall conclusion of the workshop was that the concept of integrated imaging is undervalued in the geophysical community despite applicability throughout the exploration and production life cycle, applicability in diverse geologic environments, numerous case studies, and demonstrated added value. This false perception might be addressed through more awareness using case studies illustrating the value of integrating seismic data with potential fields and other geophysical methods. To that end, a call for papers has been announced for a special section titled "Integrated geophysical imaging" in Interpretation with the goal of collecting new case studies benefiting from combining multiple geophysical methods. We anticipate publication of the Interpretation special section in August 2020.

\section{References}

Abbas, M.A., R. Calabro, D. Di Biase, M. Fedi, B. Garcea, P. Iacone, M. Mazzarelli, and L. Speranze, 2018, High resolution imaging of salt domes through integrated seismic and magnetic modelling in the east Mediterranean offshore: Presented at the 88th Annual International Meeting, SEG.

Biegert, E. K., and P. S. Millegan, 1998, Beyond recon: The new world of gravity and magnetics: The Leading Edge, 17, no. 1, 41-42, https://doi.org/10.1190/1.1437814.

Blaizot, M., and P. Montagnier, 2013, Broadening geophysics to new horizons: 83rd International Annual Meeting, SEG, Expanded Abstracts, 4478-4482, https://doi.org/10.1190/segam2013-1467.1.

Colombo, D., M. Mantovani, M. Sfolciaghi, P. van Mastrigt, A. Al-Duliajan, and T. Nafie, 2010, Near surface solutions in South Rub Al-Khali, Saudi Arabia applying seismic-gravity joint inversion and redatuming: First Break, 28, no. 2, 77-84.

Dejong, H., I. Al-Kindy, and E. Biegert, 2001, Gravity-gradient data integrated in pre-stack depth migration in the southern North Sea: 63rd Conference and Exhibition, EAGE, Paper IG-3.

Dyer, N., 2011, Using gravity gradiometry to explore subsalt: Offshore Magazine, 71, no. 1. 
Edman, J., E. Sprunt, J. Newman, M. Ruder, and J. Ellis, 2015, More efficient and cost-effective ways of evaluating and high grading unconventional plays: Interpretation, 3, no. 3, SU33-SU46, https:// doi.org/10.1190/INT-2014-0279.1.

Filina, I., N. Delebo, G. Mahapatra, C. Coble, G. Harris, J. Layman, M. Strickler, and J.-P. Blangy, 2015, Integration of seismic and gravity data-A case study from the western Gulf of Mexico: Interpretation, 3, no. 4, SAC99-SAC106, https://doi.org/10.1190/INT-2015-0050.1.

Gibson, R. I., and P. S. Millegan, 1998, Geologic applications of gravity and magnetics: SEG and AAPG, https://doi.org/10.1190/1.9781560801832.

Johnson, E. A. E., 1998, Gravity and magnetic analyses can address various petroleum issues: The Leading Edge, 17, no. 1, 98, https://doi.org/10.1190/1.1437844.

Key, K. W., S. C. Constable, and C. J. Weiss, 2006, Mapping 3D salt using the 2D marine magnetotelluric method: Case study from Gemini Prospect, Gulf of Mexico: Geophysics, 71, no. 1, B17-B27, https://doi.org/10.1190/1.2168007.

Popper, K. R., 2003, Conjectures and refutations: The growth of scientific knowledge: Routledge.

Qi, Z., X. Li, Q Zhi, H. Li, and J. Zhou, 2018, Kirchhoff migration imaging method based on groundedsource TEM virtual wavefield: Presented at the 88th Annual International Meeting, SEG.

Rauch-Davies, M., 2018, Applied use of grav mag data to delineate increased maturity in unconventional plays: Presented at the 88th Annual International Meeting, SEG.

Sheriff, R. E., 2002, Encyclopedic dictionary of applied geophysics: SEG, https://doi.org/10.1190/1 .9781560802969 .

Watson, J., J. Barraud, F. Assouline, and N. Dyer, 2010, Interpretation of gravity gradiometry data and integration with PSDM workflow-Imaging subsalt structures in Gabon: 72nd Conference and Exhibition, EAGE, Extended Abstracts, https://doi.org/10.3997/2214-4609.20149923.

Yalamanchili, R., J. Feijth, and J. Hope, 2018, Effective use of potential field and seismic methods to reduce exploration costs and ambiguity in multiple integrated imaging interpretations: 88th Annual International Meeting, SEG, Expanded Abstracts, 5458-5462, https://doi.org/10.1190/segam2018w8-01.1.

Zhdanov, M. S., 2018, New approaches to integrated imaging of multimodal geophysical data: Presented at the 88th Annual International Meeting, SEG. 